\title{
Effect of a point impurity on the vortex bound states in an $s$-wave superconductor: A self-consistent analysis
}

\author{
Qiang Han \\ Department of Physics, Peking University, Beijing 100871, China \\ and Department of Physics, University of Hong Kong, Pokfulam Road, Hong Kong, China \\ Li-yuan Zhang \\ Department of Physics, Peking University, Beijing 100871, China \\ Z. D. Wang \\ Department of Physics, University of Hong Kong, Pokfulam Road, Hong Kong, China
}

(Received 2 February 2000; revised manuscript received 12 April 2000)

\begin{abstract}
On the basis of self-consistent numerical solution of the Bogoliubov-de Gennes equations on a finite-size lattice, we study the variation of the vortex bound state when an impurity potential is added to the core of an isolated vortex line in an $s$-wave superconductor. The local density of states is investigated at both the core and its neighbor site. By analyzing the impurity-induced increase of the pair potential near the vortex core, we elucidate the mechanism of the vortex pinning in detail.
\end{abstract}

In recent years, the vortex physics of type-II superconductors has drawn much attention both theoretically and experimentally. One of the most fascinating properties of the vortex state is the quasiparticle bound states localized in the vortex cores, which were predicted decades ago by Caroli, de Gennes, and Matricon ${ }^{1}$ by solving the Bogoliubov-de Gennes (BdG) equations. ${ }^{2}$ Yet it was not until recently in 1989 that the experimental confirmation of such quasiparticle state was observed in the vortex core of $\mathrm{NbSe}_{2}$ by Hess et $a .^{3}$ who applied the scanning-tunneling microscope (STM) technique for measuring the differential tunneling conductance. Triggered by the experimental progress, selfconsistent numerical methods to solve the BdG equations have been developed on the context of both continuum ${ }^{4}$ and lattice models. ${ }^{5,6}$ All these theoretical efforts have been applied to study the electronic properties for both isolated vortex $^{4,5}$ and vortex line lattice, ${ }^{6}$ attempting to explain the experimental data. Recently, the numerical techniques have been extended to the case of $d$-wave ${ }^{6-8}$ high- $T_{c}$ cuprates and interesting features different from the conventional $s$-wave superconductors have been predicted.

On the other hand, the problem of vortex pinning has been attracting great theoretical and practical interest. For conventional $s$-wave superconductors, a traditional mechanism assumes that a void pins a vortex by attracting the vortex core in order to avoid loss of condensation energy. ${ }^{9}$ This effect is dominant for defects of large size. For the case of small defects, Thuneberg et al. ${ }^{10}$ established a microscopic theory and found how small pinning centers can be taken into account by adding extra terms to the GinzburgLandau (GL) free-energy functional. However, the conclusions obtained from the GL theory are restricted to the vicinity of the critical temperature $T_{c}$. In the present work, we report a microscopic study of the interaction between a point impurity and an isolated vortex line by applying the $\mathrm{BdG}$ theory. By analyzing the effect of the impurity on the vortex bound state and the pair potential, we obtain some interesting results about the quasiparticle scattering and vortex pinning, especially at lower temperatures where the GL description may no longer be appropriate.

The system we study is an isolated vortex line in a simple tight-binding model with an $s$-wave pairing interaction on a two-dimensional (2D) lattice with the impurity located at any given site. The model Hamiltonian can be written as ${ }^{11}$

$H=-t \sum_{\langle i j\rangle \sigma} c_{i \sigma}^{\dagger} c_{j \sigma}-\mu \sum_{i \sigma} n_{i \sigma}-V_{s} \sum_{i} n_{i \uparrow} n_{i \downarrow}+\sum_{i \sigma} V_{i}^{I m p} n_{i \sigma}$,

where $n_{i \sigma}=c_{i \sigma}^{\dagger} c_{i \sigma}$ is the electron number operator on site $i$, $\langle i j\rangle$ denotes nearest-neighbor pairs in the lattice, and $\mu$ is the chemical potential. $V_{s}>0$ is the on-site attractive potential, which gives rise to the pairing in the $s$-wave channel. $V_{i}^{I m p}$ is the impurity potential at the site $i$; for simplicity, we will study a single impurity with a $\delta$-function potential; i.e., $V_{i}^{I m p}$ is finite on some given site and zero on other sites.

By defining a mean-field pair potential $\Delta_{s}\left(\mathbf{r}_{i}\right)$ as

$$
\Delta_{s}\left(\mathbf{r}_{i}\right)=V_{s}\left\langle c_{i \downarrow} c_{i \uparrow}\right\rangle,
$$

with $\langle\cdots\rangle$ denoting the thermodynamic average, and performing the Bogoliubov transformation

$$
\left(\begin{array}{c}
c_{i \uparrow} \\
c_{i \downarrow}^{\dagger}
\end{array}\right)=\sum_{n} \gamma_{n \uparrow}\left[\begin{array}{l}
u_{n}\left(\mathbf{r}_{\mathbf{i}}\right) \\
v_{n}\left(\mathbf{r}_{\mathbf{i}}\right)
\end{array}\right]-\gamma_{n \downarrow}^{\dagger}\left[\begin{array}{c}
v_{n}^{*}\left(\mathbf{r}_{\mathbf{i}}\right) \\
-u_{n}^{*}\left(\mathbf{r}_{\mathbf{i}}\right)
\end{array}\right]
$$

with $u_{n}, v_{n}$ the quasiparticle amplitudes and $\gamma_{n \sigma}$ the quasiparticle operator, the eigen-problem of the Hamiltonian (1) can be solved from the following $\mathrm{BdG}$ equations: ${ }^{2,11}$

$$
\left(\begin{array}{cc}
\hat{\xi} & \hat{\Delta} \\
\hat{\Delta}^{*} & -\hat{\xi}^{*}
\end{array}\right)\left(\begin{array}{l}
u_{n} \\
v_{n}
\end{array}\right)=E_{n}\left(\begin{array}{l}
u_{n} \\
v_{n}
\end{array}\right),
$$

where 


$$
\begin{gathered}
\hat{\xi} u_{n}\left(\mathbf{r}_{i}\right)=-t \sum_{\tau} u_{n}\left(\mathbf{r}_{i}+\tau\right)+\left(V_{i}^{I m p}-\mu\right) u_{n}\left(\mathbf{r}_{i}\right), \\
\hat{\Delta} v_{n}\left(\mathbf{r}_{i}\right)=\Delta_{s}\left(\mathbf{r}_{i}\right) v_{n}\left(\mathbf{r}_{i}\right),
\end{gathered}
$$

and $\Delta_{s}\left(\mathbf{r}_{i}\right)$ is obtained by the self-consistent condition

$$
\Delta_{s}\left(\mathbf{r}_{i}\right)=-V_{s} \sum_{n} u_{n}\left(\mathbf{r}_{i}\right) v_{n}^{*}\left(\mathbf{r}_{i}\right) \tanh \left(E_{n} / 2 k_{B} T\right) .
$$

In the above equations, $\tau= \pm \hat{x}, \pm \hat{y}$ with the lattice constant taken to be unity and therefore $\mathbf{r}_{i}+\tau$ are the four nearestneighbor sites of site $i ; E_{n}$ is the eigenvalue of the quasiparticle state $n$ of the Hamiltonian (1).

In the study of the isolated vortex state, the boundary condition will be different from that of the homogeneous case because of the phase winding of the pair potential. Both open boundary condition and modified periodic boundary condition have been employed. For the former condition, the pair potential varies not smoothly along the boundary because quasiparticles are forbidden from hopping outwards. Therefore we apply the modified periodic boundary condition for improvement. To construct modified periodic boundary conditions for $u_{n}, v_{n}$ properly, a lattice area accommodating at least one electronic flux quantum $\phi_{0}=h c / e$ (thus two superconducting flux quanta $\Phi_{0}=h c / 2 e$ ) must be used because $u_{n}$ and $v_{n}$ describe the behaviors of the quasielectron and quasihole, respectively, which have charge $\pm e .^{6}$ We emphasize that the existence of two vortices will not have remarkable effects on the vortex bound states of each other as long as the two cores are separated far enough. In this paper, the lattice consists of two connected square areas, each containing a vortex core at the center as shown in Fig. 1(a). From now on, the lattice site $\mathbf{r}_{i}$ will be represented by $(p, q)$ ( $p, q$ are integers), with one of the two vortex cores sitting on $(0,0)$. The system size is $(2 L+1) \times(4 L+1)$, with $L$ half of the width of the lattice. We choose a special gauge as in Ref. 12, where $\Delta_{s}$ satisfies the condition

$$
\begin{gathered}
\Delta_{s}(L, q)=\Delta_{s}(-L, q) \exp (i 2 \pi q / L), \\
\Delta_{s}(p, 3 L)=\Delta_{s}(p,-L),
\end{gathered}
$$

with the corresponding boundary conditions for $u_{n}$ and $v_{n}$ :

$$
\begin{gathered}
u_{n}(L, q)=-u_{n}(-L, q) \exp (i \pi q / L), \\
v_{n}(L, q)=-v_{n}(-L, q) \exp (-i \pi q / L), \\
u_{n}(p, 3 L)=-u_{n}(p,-L), \\
v_{n}(p, 3 L)=-v_{n}(p,-L) .
\end{gathered}
$$

With the above boundary conditions, the resulting quasiparticle-excitation spectrum is obtained by repeated diagonalization of the Hamiltonian and iteration of the pair potential. The BdG Eq. (4) is studied under the following set of parameters: The size of the lattice is $17 \times 33(L=8) ; \mu$ $=-2 t$ (with the band bottom at $-4 t$ ) gives rise to a band filling factor $\langle n\rangle \simeq 0.38 ; V_{s}=3 t$ corresponds to the bulk value of the pair potential $\Delta_{0} \simeq 0.45 t$ at zero temperature and $T_{c} \simeq 0.26 t$. The system temperature is set to $0.01 t$. In Fig. 1(b), the spatial variation of the amplitude of the pair poten- (a)

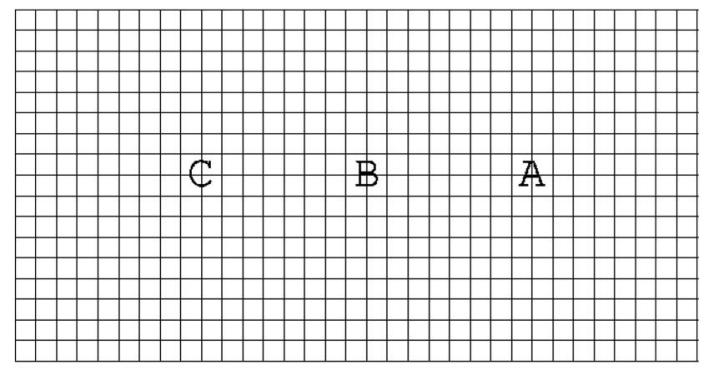

(b)

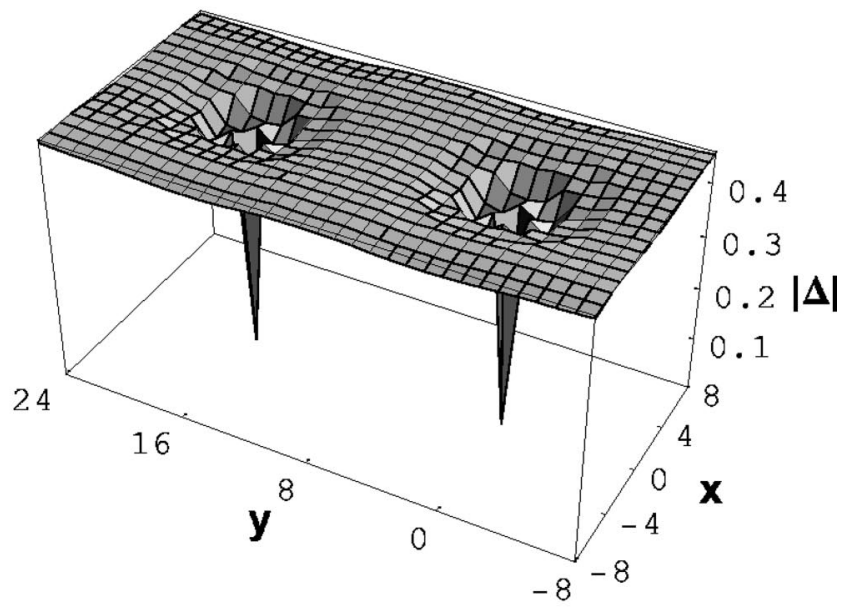

FIG. 1. (a) Illustration of the $(2 L+1) \times(4 L+1)$ lattice containing two vortices with cores denoted by $\mathrm{A}$ and $\mathrm{C}$, one of which, $\mathrm{A}$, is selected as the origin. Site B is at the middle of the two cores; (b) spatial variation of $\left|\Delta_{s}\right|$.

tial $\Delta_{s}$ is shown. It is seen that over 2-4 lattice spaces $\Delta_{s}$ reaches its bulk value indicating that the coherence length $\xi$ is of the order of lattice constance $a$, which is consistent with an estimate obtained from the BCS formula $\xi=\hbar v_{F} / \pi \Delta_{0}$ $\sim E_{F} / \Delta_{0} k_{F} \sim 4 k_{F}^{-1} \sim 2 \sqrt{2} a$. The short $\xi$ results in an unequally spaced energy levels of the bound states. We find that the distribution of the lowest seven bound-state eigenvalues nomalized by $\Delta_{0}$ is $E / \Delta_{0}=0.22,0.43,0.53,0.60$, $0.66,0.72,0.76$, which is quite different from the constant spaced energy levels found in Ref. 1 where $\xi \gg k_{F}^{-1}$ was assumed, but in good agreement with the results of the continuum model ${ }^{13}$ when $4 k_{F}^{-1}<\xi<8 k_{F}^{-1}$. Figure 2 shows the spatial variation of the quasiparticle amplitudes $|u|^{2}$ and $|v|^{2}$ corresponding to the lowest bound state, which indicates strong localization around the vortex core. $|u|^{2}$ has a high peak at the site $(0,0)$ and decays exponentially over a distance of $\xi ;|v|^{2}$ has zero amplitude at the vortex center and high peaks at the $(1,0)$ and $(1,1)$ sites. For higher boundstate energy levels, $|u|^{2}$ and $|v|^{2}$ behave similarly except that the site positions of their high peaks move farther from the central site $(0,0)$. Furthermore, of all the vortex bound state, only the $|u|^{2}$ corresponding to the lowest bound state with eigenvalue $E / \Delta_{0}=0.22$ has nonzero amplitude at the site $(0,0)$, which leads to the high peak structure of local density of states (LDOS) at the vortex core and its sensitivity to the existence of the impurity sitting on the core site as discussed below. 

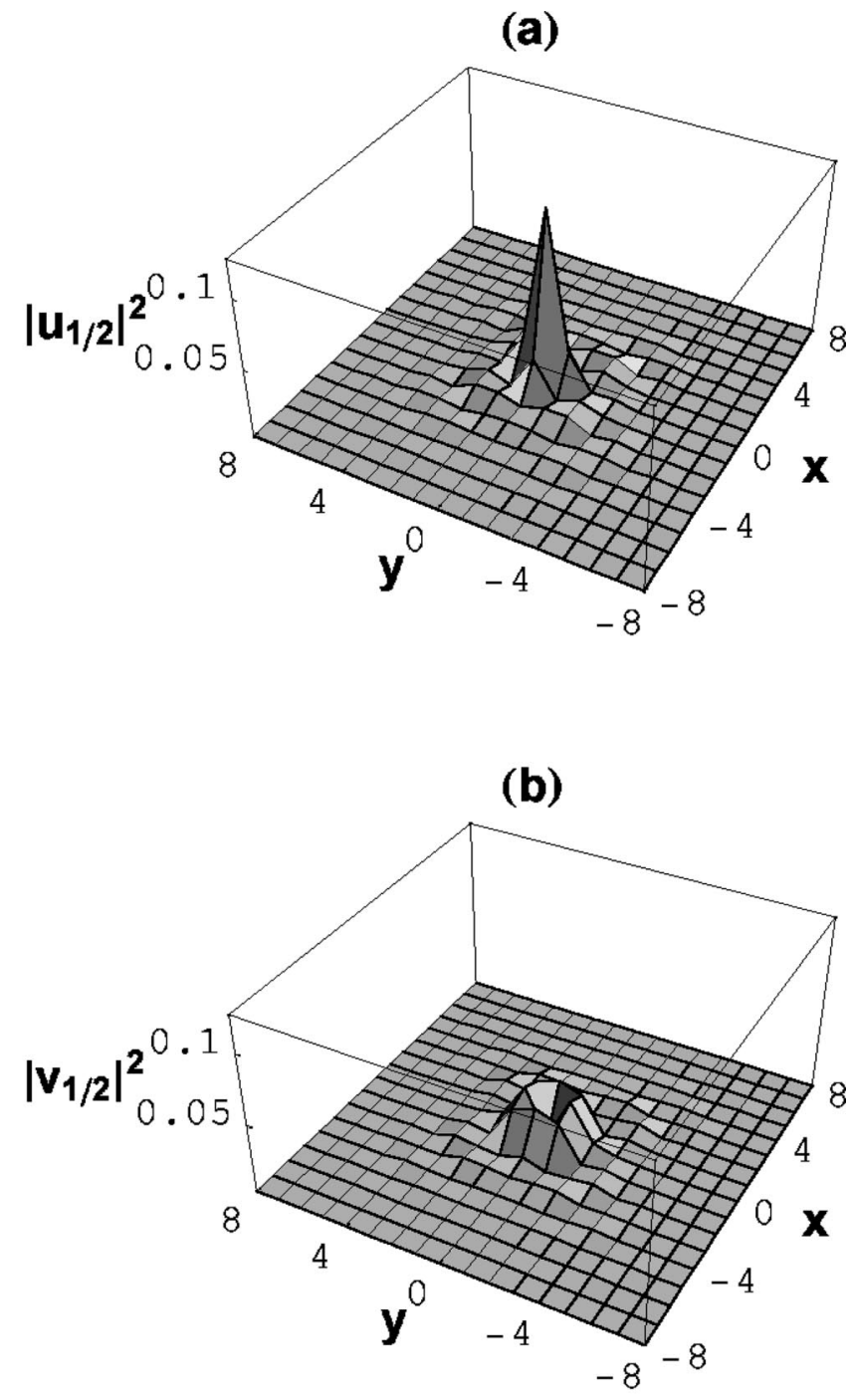

FIG. 2. Spatial distribution of the quasiparticle amplitudes $|u|^{2}$ and $|v|^{2}$ within one square lattice area corresponding to the lowest bound state with eigenvalue $E=0.10 t$.

First, we consider the impurity-induced variation of the low-lying excitations around the vortex core. The local density of states (LDOS) defined by

$$
\begin{aligned}
N(p, q ; E)= & \sum_{n}\left[\left|u_{n}(p, q)\right|^{2} f^{\prime}\left(E-E_{n}\right)\right. \\
& \left.+\left|v_{n}(p, q)\right|^{2} f^{\prime}\left(E+E_{n}\right)\right]
\end{aligned}
$$

is studied, which is proportional to the differential tunneling conductance of the scanning tunnel microscope (STM) experiments. Here, $f(E)$ represents the usual Fermi distribution function. For a simpler case with $V^{I m p}=0$, Fig. 3(d) shows the LDOS at the vortex center $(0,0)$. Clearly, there is a high LDOS peak right above the Fermi level at about $0.10 t\left(0.22 \Delta_{0}\right)$, arising from the lowest bound state. Within the energy gap $\Delta_{0}$, this is the only LDOS peak at the vortex core because the quasiparticle amplitude $|u(0,0)|^{2}$ of other bound states vanish as stated above. On the site $(1,0)$, there are four LDOS peaks, which arise from the contributions of $|u(1,0)|^{2}$ and $|v(1,0)|^{2}$ of the bound states with eigenvalues $E=0.10 t$ and $E=0.19 t$. At $E=0.10 t$, the height of the

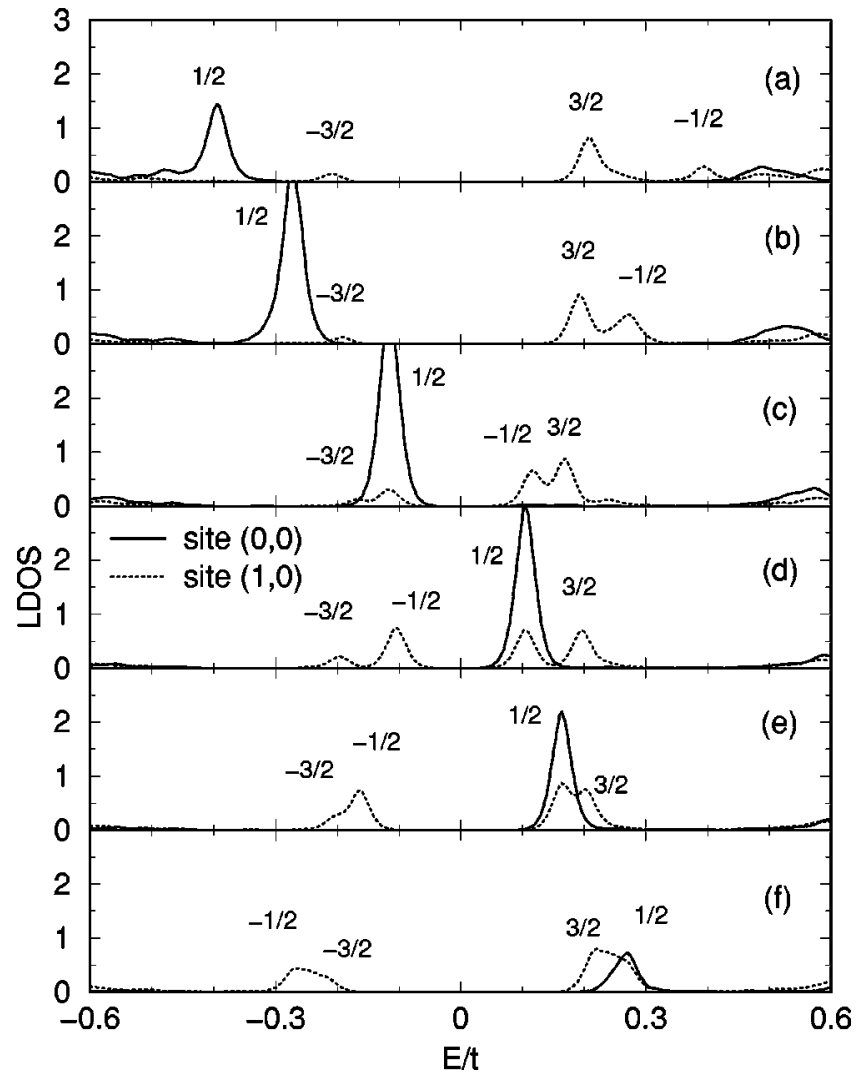

FIG. 3. LDOS at $T=0.01 t$ on site $(0,0)$ (solid line) and $(1,0)$ (dotted line). From (a) to (f), $V^{I m p}=-3 t,-2 t,-1 t, 0,0.5 t, 2 t$, respectively.

LDOS peak at site $(1,0)$ is only about $23 \%$ of that at site $(0,0)$ due to the rapid decay of $|u|^{2}$ as shown in Fig. 2(a). Above $\Delta_{0}$, the LDOS is finite owing to the contribution from the extended states. All these results are qualitatively consistent with those of the continuum model. ${ }^{13}$ Although for our discrete lattice geometry, the cylindrical symmetry is broken, yet fourfold rotation symmetry can still be employed to label the core states conveniently. As previously pointed out in Ref. 5, when the fourfold rotational symmetry of the underlying lattice is taken into account, the quasiparticle amplitudes have the following relations under an elementary $\pi / 2$ rotation:

$$
\left[\begin{array}{l}
u(-q, p) \\
v(-q, p)
\end{array}\right]=\left[\begin{array}{l}
u(p, q) e^{i(\nu-1 / 2) \pi / 2} \\
v(p, q) e^{i(\nu+1 / 2) \pi / 2}
\end{array}\right],
$$

where $\nu= \pm 1 / 2, \pm 3 / 2$ corresponds to the four irreducible representations of the $C_{4}$ rotation group. Therefore we can conveniently classify the vortex core bound state according to its $\nu$. Similar to the continuum model, we find that for the $\nu=1 / 2$ states $u_{1 / 2}(0,0) \neq 0, v_{1 / 2}(0,0)=0$ and for the $\nu=$ $-1 / 2$ states $u_{-1 / 2}(0,0)=0, v_{-1 / 2}(0,0) \neq 0$, which implies a finite probability of the quasiparticle appearing at the core; while for the quasiparticle excitations with labels $\pm 3 / 2, \quad u_{ \pm 3 / 2}(0,0)=v_{ \pm 3 / 2}(0,0)=0$. We find that the lowest bound state has $\nu=1 / 2$ and eigenvalue $E_{1 / 2}=0.10 t$ and it contributes to the high LDOS peak at $0.10 t$ as shown in Fig. 3 (d); its time-reversal counterpart has $\nu=-1 / 2$ and $E_{-1 / 2}$ $=-E_{1 / 2}$. Due to its much greater probability at the core, the lowest bound state will be more sensitive to the existence of 


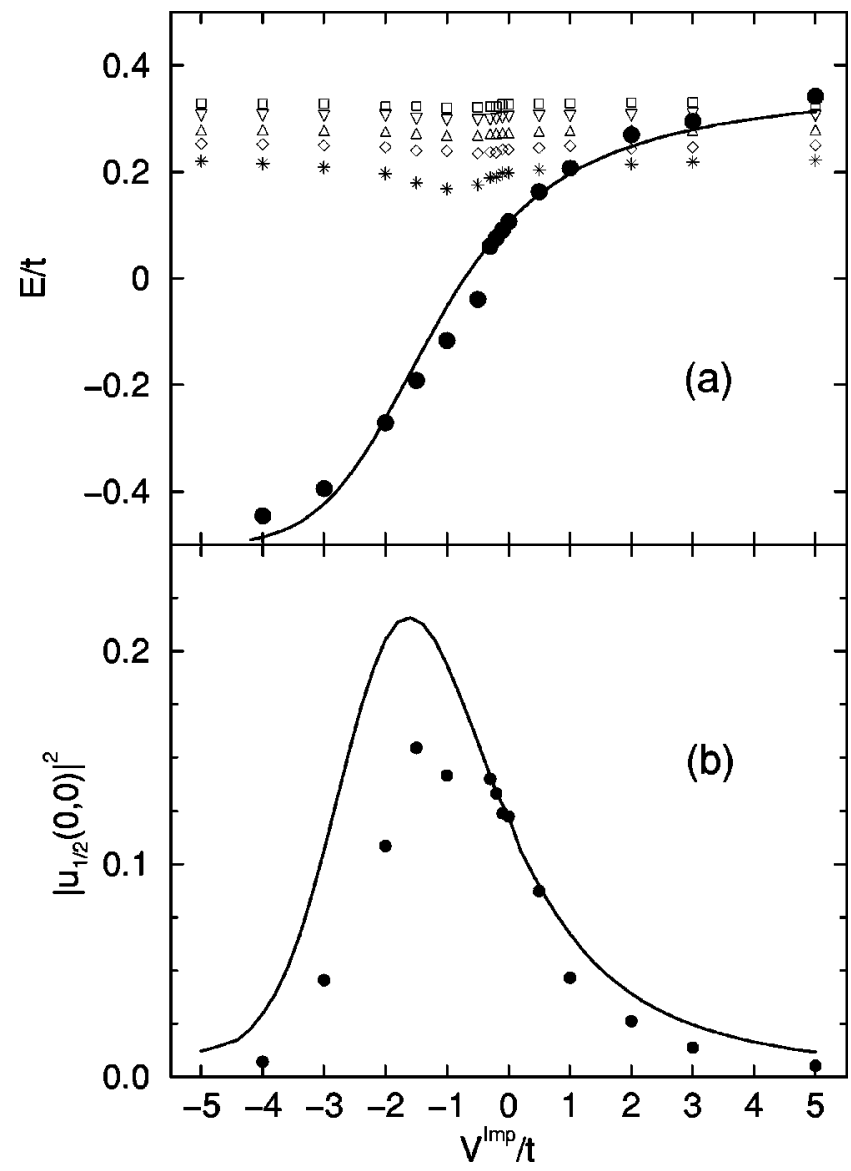

FIG. 4. Variation of the lowest six bound state with $V^{\operatorname{Imp}}$. (a) shift of the eigenvalues $\mathrm{E}$ versus $V^{I m p}$. Filled circles, stars, diamonds, up triangles, down triangles, and open squares correspond to the bound states with eigenvalues $E / t=0.10,0.19,0.24,0.27$, $0.30,0.32$, respectively, when $V^{\operatorname{Imp}}=0$; (b) $\left|u_{1 / 2}(0,0)\right|^{2}$, versus $V^{I m p}$. The filled circles denote the results from the self-consistent calculation while the lines from approximate method.

impurity potential than other states. Figure 3 shows how first few bound states are affected when $V^{I m p} \neq 0$. For an increasing repulsive impurity potential, $V^{\text {Imp }}>0$, Figs. 3(d)-(f) indicate that the core state is "repulsed" by the impurity to higher level away from the Fermi energy; its time-reversal state shifts downward accordingly satisfying the relation $E_{-1 / 2}=-E_{1 / 2}$. Together with the lifting of eigenvalue, the LDOS peak at the core lowers. The shifting of the eigenvalue and decreasing of the LDOS can also be seen for high-energy extended states above $\Delta_{0}$. The bound states with $\nu= \pm 3 / 2$ have no remarkable variation, which is consistent with the fact that their wave functions have zero amplitude at the vortex center. For the case where $V^{I m p}<0$, the results are a little complex. As shown in Figs. 3(a)-(d), with the descending of the eigen-energy, the LDOS peak first increases for weak attractive potential and then reduces for strong enough $V^{I m p}$. Figure 4(a) gives the variation of the lowest six boundstate eigenvalues as a function of $V^{I m p}$. Clearly, the vortex core state with $E_{1 / 2}=0.10 t$ varies strongly while the other bound states with higher energies keep almost constant. ${ }^{14}$ When $\left|V^{\operatorname{Im} p}\right| \gg t$, a localized impurity state will appear with $|u(0,0)| \approx 1$; for $V^{\operatorname{Im} p}>0$, it is a hole state below the Fermi level while for $V^{I m p}<0$, it is an electron state above the Fermi level. Therefore all the nonzero $u(0,0)$ belonging to

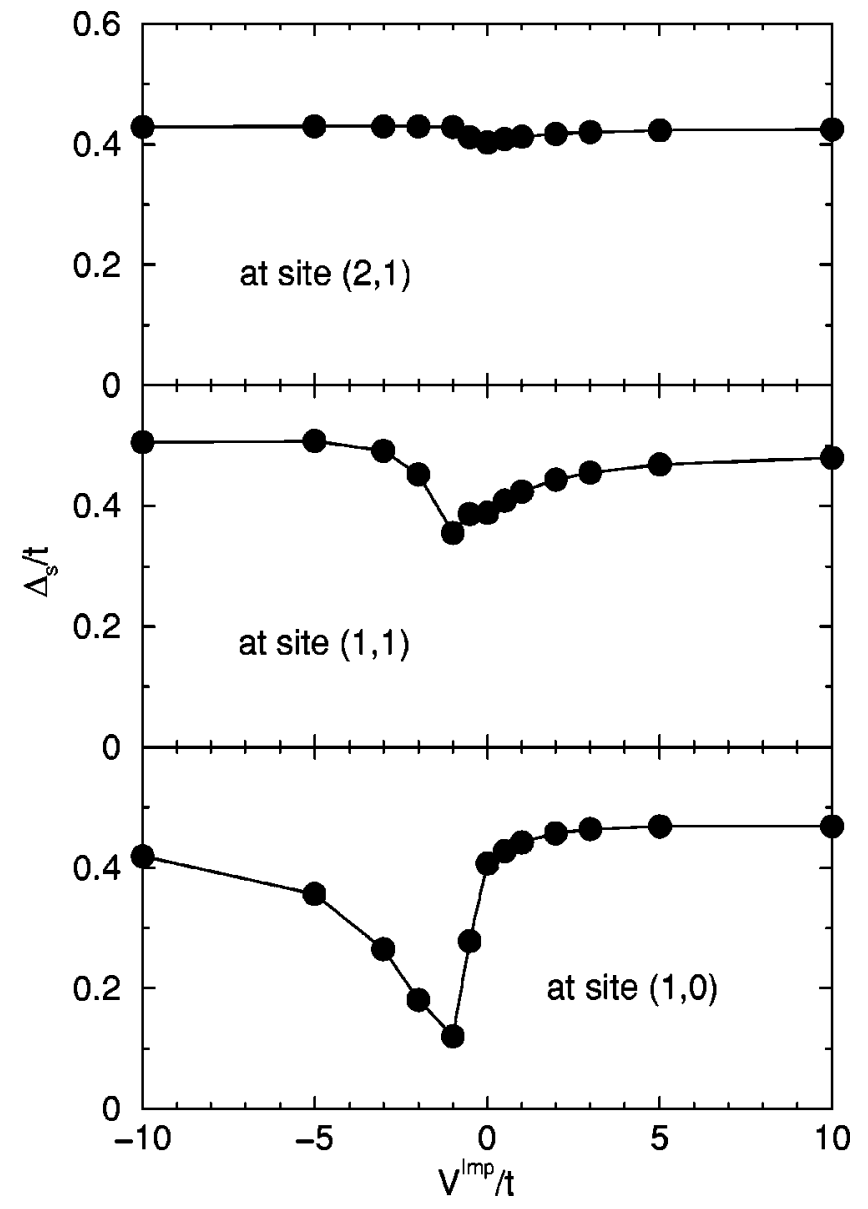

FIG. 5. Variation of the pair potential $\Delta_{s}$ with $V^{\text {Imp }}$ at the neighbor sites of the vortex core, $(1,0),(1,1)$, and $(2,0)$. The lines are guides for eyes.

other states will be absorbed by the impurity state and approach to zero for the large $V^{\text {Imp }}$ limit. The abnormal behavior of the LDOS for weak attractive $V^{\text {Imp }}$ is attributed to the asymmetric distribution of the $\nu=1 / 2$ states below and above the Fermi level. In our case where $\mu=-2 t$, there are more $\nu=1 / 2$ states above the Fermi level than those below. To examine our thought that the impurity potential affects mainly the states which have larger probability at the core, we have performed an approximate computation in which only excitations with $|u(0,0)| /|u(0,0)|_{\text {max }} \geqslant 0.01$ are included. The comparison of the results of such approximate method with those of the self-consistent calculation is shown in Fig. 4. For $V^{\operatorname{Im} p}>0$, we find that the approximate method can well describe the lifting of $E_{1 / 2}$ and the reducing of $\left|u_{1 / 2}(0,0)\right|^{2}$ with the increasing of the intensity of the impurity potential. For $V^{I m p}<0$, the discrepancy is large, yet qualitative trend still can be given.

The straightforward consequence of the impurity-induced effect on the quasiparticle excitations at the vortex core will be the variation of the pair potential at the neighbor sites. From Fig. 5 we see that for large $V^{\operatorname{Im} p}, \Delta_{s}$ at the neighbor sites rises and such rising of the pair potential is only remarkable at the nearest neighbors: $(1,0),(1,1)$ and their equivalent sites. As discussed above, for the $\nu= \pm 1 / 2$ states, either $u(0,0)$ or $v(0,0)$ of the two quasiparticle amplitudes will be zero at the vortex core, which results in the other nonzero one "useless" to the formation of $\Delta_{s}$ according to 
Eq. (5). After an impurity potential is added to the core, the unused amplitude will be squeezed out to the neighbor sites which gives rise to the increasing of $\Delta_{s}$ at these sites. And this mechanism can also be used to explain the reducing of $\Delta_{s}$ for weak attractive $V^{I m p}$ now that $|u(0,0)|$ increases as shown in Fig. 4(b). The variation of $\Delta_{s}$ near the vortex center can be used to explain the weakness of the above approximate method in quantitatively describing the behavior of the $E_{1 / 2}$ bound state for weak attractive $V^{I m p}$. Due to the variation of $\Delta_{s}$, states having zero amplitudes at the vortex core will no longer be orthogonal to the $\nu=1 / 2$ states and therefore they will take part in the coupling, which makes our approximate method have larger discrepancy when the $\Delta_{s}$ varies greatly for $V^{\operatorname{Im} p} \sim-t$.

At last, we discuss the pinning energy $E_{\text {pinning }}$ which is the energy saved when the vortex core is located at the impurity site. $E_{\text {pinning }}$ is defined as

$$
\begin{aligned}
E_{\text {pinning }} & =\langle H\rangle_{\text {impurity at core }}-\langle H\rangle_{\text {impurity at } \infty} \\
& \approx\langle H\rangle_{\text {impurity at site A }}-\langle H\rangle_{\text {impurity at site B }},
\end{aligned}
$$

where the sites A and B are shown in Fig. 1(a). We have approximately treated site $\mathrm{B}$ as $\infty$, which is valid when the system size is sufficiently large. $\langle H\rangle$ is given by

$$
\begin{aligned}
\langle H\rangle= & \sum_{n} E_{n} \sum_{p, q}\left\{\left|u_{n}(p, q)\right|^{2} f\left(E_{n}\right)\right. \\
& \left.-\left|v_{n}(p, q)\right|^{2}\left[1-f\left(E_{n}\right)\right]\right\} .
\end{aligned}
$$

In Fig. 6 we show the relation of $E_{\text {pinning }}$ with $V^{I m p}$. In the strong $V^{I m p}$ limit, both attractive and repulsive impurity potentials can pin vortex by lowering the average total energy. For weak attractive $V^{I m p}$, pinning at the impurity site of the vortex core is not favorable from the consideration of energy. From Fig. 6 we find that the dominant contribution to $E_{\text {pinning }}$ is from the condensation energy. As discussed above, a strong impurity potential at the vortex core can drive out the extra quasiparticle amplitudes which have no contribution to the formation of pair potential at the core to its nearby sites; on the other hand, this extra contribution will disappear when the impurity is on site $\mathrm{B}$. Therefore existence of the impurity at the core site will lower the condensation energy more than at other sites. Again, the exception is for weak negative $V^{I m p}$ where the pair potential is somewhat destructed due to the attraction of the wave amplitude from the neighbor sites to the core site. Moreover, Fig. 6 indicates that the supercurrent kinetic energy increases with $V^{\operatorname{Imp}}$ which is not in favor of the pinning. These results are consistent with those from the GL theory ${ }^{15}$ where the enhancement of the pair potential and supercurrent near the pinning center is also found.

In summary, we have analyzed a pinning problem by solving the BdG equations self-consistently. We have found

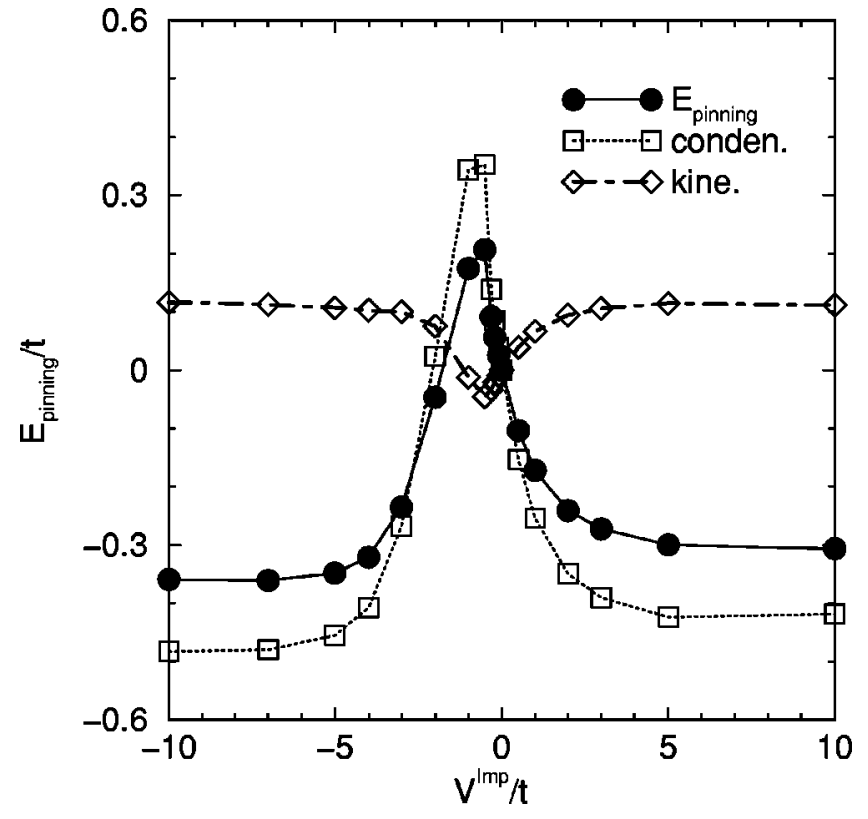

FIG. 6. Pinning energy $E_{\text {pinning }}$ as a function of $V^{I m p}$. The contributions from the condensation energy (conden.) and the supercurrent kinetic energy (kine.) are plotted as well.

that an impurity potential at the vortex core can drive the lowest bound state away from the Fermi level to higher energy. At the same time, its LDOS at the vortex core reduces. Although these results are obtained for superconductors with short coherence length, yet we believe the conclusion can still be applied qualitatively to $s$-wave superconductors with longer coherence length such as $\mathrm{NbSe}_{2}\left(\xi \sim 70 k_{F}^{-1}\right)$ and $\mathrm{V}_{3} \mathrm{Si}\left(\xi \sim 12 k_{F}^{-1}\right),{ }^{13}$ when the temperature is low enough. Recent progress ${ }^{16,17}$ in atomic-scale STM measurements might make the experimental observation of such impurityinduced vortex bound state shifting feasible. Atomic scale defects can be deposited onto the surface of the superconducting sample right from the STM tip. If a single vortex line could be successfully pinned by such defects, the observation of the variation of the LDOS at the vortex core as a function of impurity potential strength will be easier by introducing different impurities from the STM tips made of Au, W, etc. For the mechanism of vortex pinning, we have shown how an impurity potential increases the condensation energy by driving the extra density of states at the vortex core into its nearby sites resulting in the increase of the pair potential at such sites.

This work was supported by the RGC grant of Hong Kong under Nos. HKU 7116/98P and HKU 7144/99P, a CRCG grant at the University of Hong Kong, and a grant from the National Natural Science Foundation of China. 
${ }^{1}$ C. Caroli, P.G. de Gennes, and J. Matricon, Phys. Lett. 9, 307 (1964).

${ }^{2}$ P. G. de Gennes, Superconductivity of Metals and Alloys (Benjamin, New York, 1966).

${ }^{3}$ H.F. Hess, R.B. Robinson, R.C. Dynes, J.M. Valles, Jr., and J.V. Waszczak, Phys. Rev. Lett. 62, 214 (1989).

${ }^{4}$ F. Gygi and M. Schlüter, Phys. Rev. B 43, 7609 (1991).

${ }^{5}$ Y.D. Zhu, F.C. Zhang, and M. Sigrist, Phys. Rev. B 51, 1105 (1995).

${ }^{6}$ Y. Wang and A.H. MacDonald, Phys. Rev. B 52, R3876 (1995).

${ }^{7}$ Y. Morita, M. Kohmoto, and K. Maki, Phys. Rev. Lett. 78, 4841 (1997).

${ }^{8}$ M. Franz and Z. Tesǎnović, Phys. Rev. Lett. 80, 4763 (1998).

${ }^{9}$ A.M. Campbell and E. Evetts, Adv. Phys. 21, 199 (1972).

${ }^{10}$ E.V. Thuneberg, J. Kurkijärvi, and D. Rainer, Phys. Rev. Lett. 48, 1853 (1982); Phys. Rev. B 29, 3913 (1984); E.V. Thuneberg, J. Low Temp. Phys. 57, 415 (1984); Cryogenics 29, 236 (1989).

${ }^{11}$ P.I. Soininen, C. Kallin, and A.J. Berlinsky, Phys. Rev. B 50, 13 883 (1994); M. Franz, C. Kallin, and A.J. Berlinsky, ibid. 54, R6897 (1996).

${ }^{12}$ Z.D. Wang and C.R. Hu, Phys. Rev. B 44, 11918 (1991); J.H.

Xu, Y. Ren, and C.S. Ting, ibid. 53, R2991 (1996).
${ }^{13}$ N. Hayashi, T. Isoshima, M. Ichioka, and K. Machida, Phys. Rev. Lett. 80, 2921 (1998).

${ }^{14}$ As pointed out later, the existence of the impurity potential gives rise to the variation of the pair potential around the vortex core and leads also to weak shifts of the higher energy levels according to Eq. (4). However, such effect is of second order and therefore the shifts are trivial. Furthermore, impurity does not change the fact that the higher energy bound states have zero quasiparticle amplitudes at the vortex core; thus we can always trace the shift of the lowest bound state by examining its nonzero quasiparticle amplitude at the vortex core. However, for sufficiently strong impurity potential, $V^{I m p}<-4 t$ or $V^{I m p}>5 t$, the tracing will fail because the lowest vortex core state shifts beyond the energy gap $\Delta_{0}$ or the quasiparticle amplitude is too suppressed to be distinguished.

${ }^{15}$ M. Friesen and P. Muzikar, Phys. Rev. B 53, R11 953 (1996); 55, 509 (1997); 57, 2709 (1998);

${ }^{16}$ E.W. Hudson, S.H. Pan, A.K. Gupta, K.-W. Ng, and J.C. Davis, Science 285, 88 (1999).

${ }^{17}$ A. Yazdani, C.M. Howald, C.P. Lutz, A. Kapitulnik, and D.M. Eigler, Phys. Rev. Lett. 83, 176 (1999). 